\title{
ALMA and the Future of Millimeter Imaging Observations
}

\author{
David J. Wilner \\ Harvard-Smithsonian Center for Astrophysics \\ email: dwilner@cfa.harvard.edu
}

\begin{abstract}
The Nearby Young Moving Groups sample the critical age when primordial disks around stars complete their transformation into planetary systems with associated debris. Millimeter wavelengths provide direct access to cool material in these circumstellar disks. The high angular resolution of interferometry at these long wavelengths enables resolved observations of solids in an optically thin regime, as well as the thermal, chemical, and dynamical structure of gas, if present. In this contribution, I briefly review the evolving landscape of millimeter telescopes, with emphasis on the revolutionary capabilities of the new international Atacama Large Millimeter/submillimeter Array (ALMA) and describe pertinent early science results.
\end{abstract}

Keywords. circumstellar matter, planet-disk interactions, submillimeter: planetary systems

\section{Introduction}

It is not an exaggeration to say that high resolution imaging observations at millimeter wavelengths are undergoing a revolution, the direct result of a decades long international effort to develop and construct the Atacama Large Millimeter/submillimeter Array (ALMA). Several other contributons in this volume discuss ALMA results, notably from Session 3 of the conference on disks orbiting Nearby Young Moving Group members, including reviews of protoplanetary disk evolution (Cieza), transitional disk structure (van der Marel), disk chemistry (Öberg), and debris disks (Kospal). In this contribution, I take a step back to review the relevance of millimeter wavelength observations for studying circumstellar disks, and then I briefly discuss the landscape of millimeter telescopes to put ALMA into context, followed by a few selected examples from ALMA early science.

\section{Millimeter Wavelength Observations of Disks}

The "millimeter" regime spans about two orders of magnitude in wavelength, from about 300 microns, limited at shorter wavelengths by insufficient atmospheric transmission to the ground, to wavelengths of a few centimeters, melding into the domain of traditional radio astronomy. Figure 1 shows the full spectral energy distribution of TW Hya, representative of a nearby young star surrounded by a circumstellar disk. The spectral energy distribution is dominated by two components, the stellar photosphere that peaks in the optical, and a broad excess above the photosphere from thermal dust emission that extends from the infrared into the millimeter. It's very obvious from Figure 1 that emission at millimeter wavelengths is not significant in an energetic sense. However, the emission at millimeter wavelengths has useful characteristics that make observations of disks in this regime important. First, the emission becomes optically thin and therefore provides a direct tracer of mass (in solids). Second, millimeter emission allow unique access to cold material, 10's of K and below, thereby sampling the bulk of the disk material, including the midplane. Third, many common molecules have spectral lines in the 


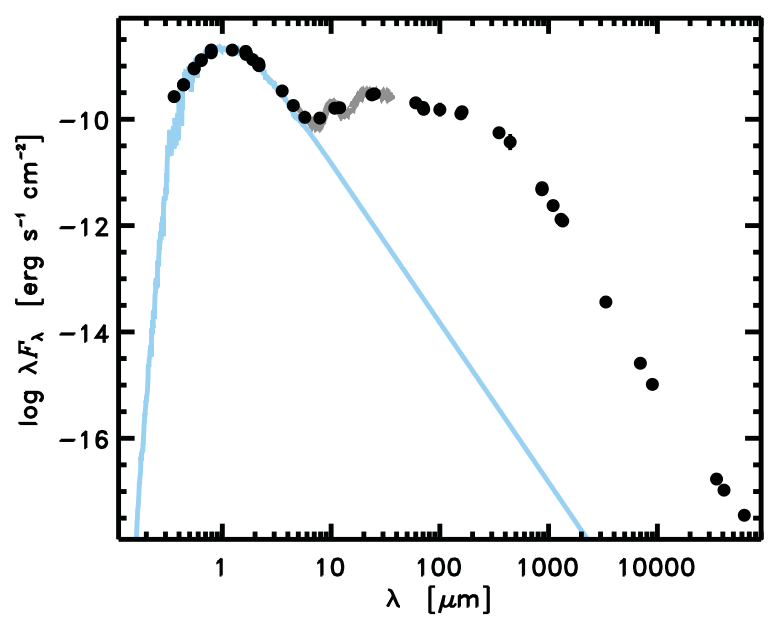

Figure 1. The spectral energy distribution of TW Hya (see Menu et al. 2014), showing a model stellar photosphere peaking in the optical (blue curve) and observations of dust emission in excess above the photosphere from the infrared through the millimeter.

millimeter, which offer probes of gas heating, cooling, and chemistry. With receivers using heterodyne techniques, the spectral resolution is naturally very high, $\Delta \lambda / \lambda>10^{6}$, enabling detailed studies of gas motions. Finally, the stellar photosphere is relatively faint at these long wavelengths and contrast with disk material is not a problem, allowing straightforward access to planet-forming regions. These characteristics are all wonderful, but the catch is that emission from cold, optically thin material has very low brightness. As a consequence, very high sensitivity is required for imaging, especially at the high angular resolution needed for circumstellar disks. This is the key reason why the enormous sensitivity gain realized by ALMA is driving a revolution.

\section{The Landscape of Millimeter Telescopes}

Figure 2 shows a schematic view of telescopes currently operating in the 1.3 millimeter atmospheric window. The circles in this Figure are scaled by the physical diameter of the telescope antennas, which serves as a proxy for sensitivity (all else being equal). ALMA is clearly a very large telescope by the standards of existing facilities. Note that ALMA includes a main array of $50 \times 12$ meter antennas, and a compact array to obtain information on larger size scales with $12 \times 7$ meter antennas and four additional 12 meter antennas for total power observations. The 66 high precision (and movable) antennas that comprise ALMA are sited at 5000 meters elevation in the Chilean Andes, above much of the atmospheric water vapor that absorbs millimeter radiation. ALMA represents an improvement by one to two orders of magnitude in essentially every way over other millimeter telescopes. Besides the enormous collecting area (equivalent to a single $\sim 80$ meter antenna), the large number of antennas results in superior interferometric image fidelity, and the main array configurations can extend to $15 \mathrm{~km}$ to provide resolutions as high as 5 milliarcseconds at the shortest wavelengths. ALMA represents a fusion of international efforts under development since the 1980 's, and is funded by an international partnership of North America (37.5\%) Europe (37.5\%), and East Asia (25\%), in cooperation with the Republic of Chile. Up-to-date details about ALMA technical capabilities and management can be found at www.almaobservatory.org. 

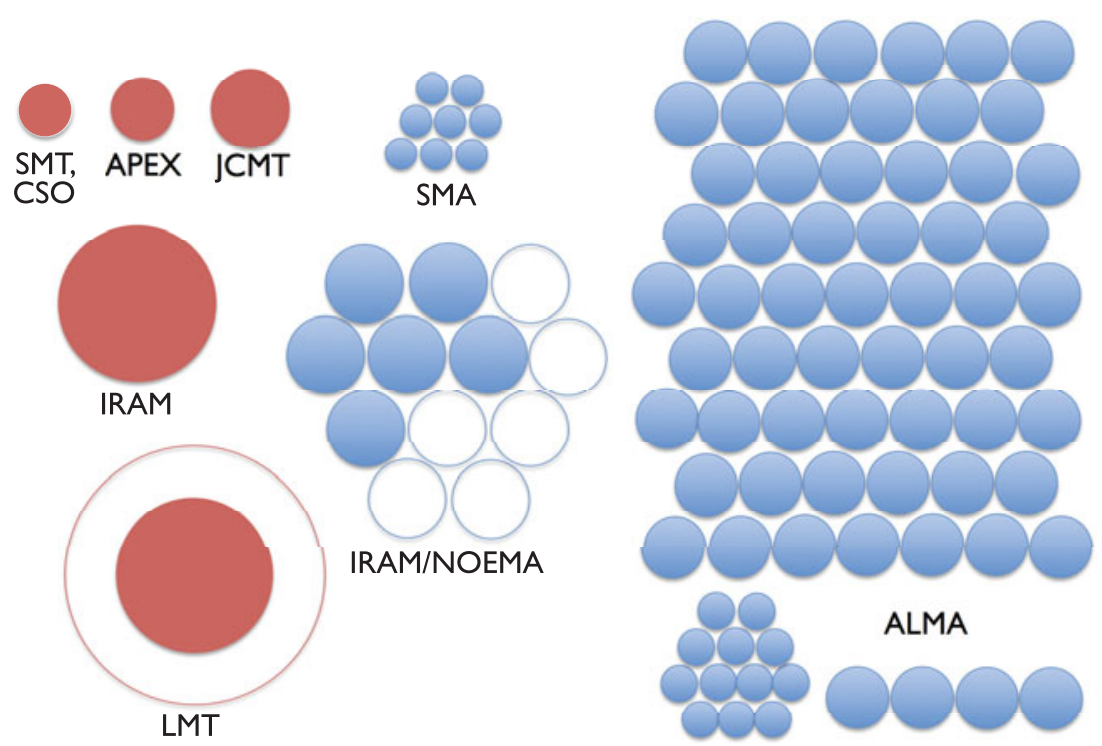

Figure 2. The landscape of millimeter telescopes. The circle size is proportional to antenna diameter. On the left, color coded in orange, are single dish telescopes; on the right, color coded in blue, are interferometers.

An improvement in observational capability this large comes along perhaps only once in the career of a typical observational astronomer. Community interest in using ALMA has been overwhelming. ALMA Cycle 0 early science began in October 2011 with just 16 antennas and angular resolutions no better than other arrays, but already the sensitivity gains were significant; 919 proposals were submitted and 113 projects selected (with time allocated according to partner share). The most recent Cycle 3 call, for operation with at least 36 antennas and baselines to $10 \mathrm{~km}$, resulted in 1592 proposals submitted, the most ever for any observatory.

\section{ALMA's Revolutionary Sensitivity and Imaging Speed}

ALMA's power allows for observations that couldn't be done before in a reasonable time. For example, a few hour ALMA observation of a source at $50 \mathrm{pc}$ distance is sensitive to dust masses less than a few tenths of a lunar mass (for standard dust opacities and disk temperatures). New statistical views of populations of sources become possible. Figure 3 shows a montage of ALMA Cycle 0 observations at 0.88 millimeters of $20 \mathrm{~K}$ and M-type stars surrounded by disks in the 5-10 Myr-old Upper Sco OB association, from Carpenter et al. 2014. Each source was observed in just a few minute snapshot, with lower noise and better imaging than could be obtained after integration times of hours using other arrays. These ALMA data were used by to measure the dust content in these disks, down to 0.3 Earth masses, with 13 detections ( 4 of which are resolved). The image of the transition disk surrounding the star J160421.7-213028 (second row, second column) is a particularly impressive (compare with the SMA observations of Mathews et al. 2012.) ALMA observations of this disk, which were also configured for the CO J=3-2 line, were further analyzed by Zhang et al. 2014 to show that $80 \%$ of the dust mass is concentrated in a $35 \mathrm{AU}$ wide annulus, while the $\mathrm{CO}$ emission extends to both substantially larger and smaller radii. The strong radial concentration of millimeter dust emission in the gas disk suggests the presence of a pressure trap, perhaps created by a planet inside the cavity. 


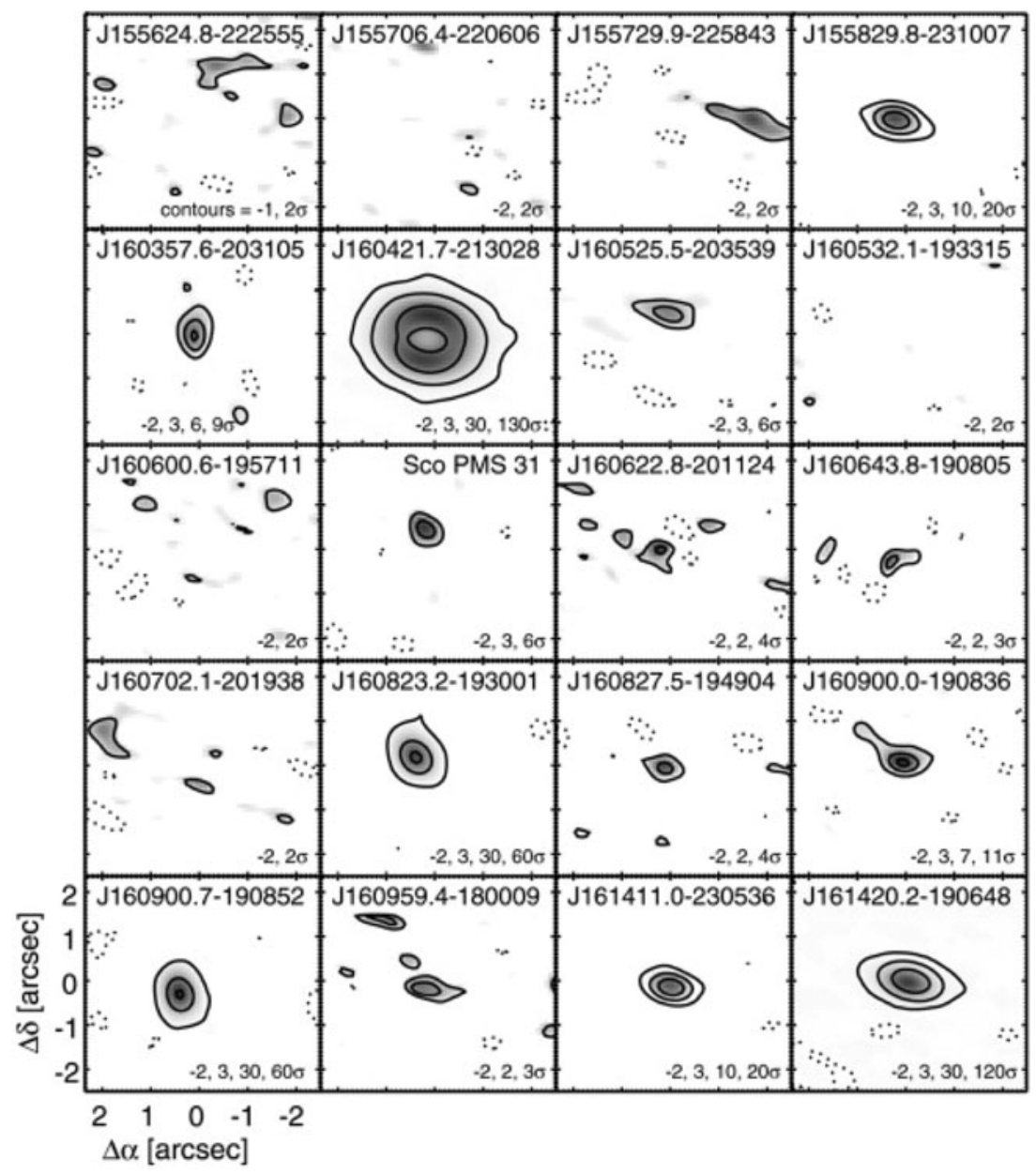

Figure 3. ALMA Cycle 00.88 millimeter snapshot images of 20 K-M type stars in Upper Sco, from Carpenter et al. 2014. The contour levels are indicated in the lower right of each panel; the $1 \sigma$ sensitivity is typically $0.2 \mathrm{mJy}^{\text {beam }}{ }^{-1}$ (beam size $\sim 0.3 \operatorname{arcsec}$ ).

A byproduct of ALMA observations of gas-rich circumstellar disks will be disk-based dynamical masses of their stellar hosts. Rosenfeld et al. 2012 present a proof of concept study of this technique using SMA observations of CO J=2-1 emission from the circumstellar disk orbiting the double-lined spectroscopic binary star V4046 Sgr, a member of the $\beta$ Pictoris moving group. Modeling the Keplerian velocity field of the disk around V4046 Sgr gives an estimate of the total mass of the central binary that is in excellent agreement with independent dynamical constraints obtained from monitoring the stellar radial velocities. This comparison in the V4046 Sgr system validates the absolute accuracy of this high precision (few percent) disk-based method for estimating stellar masses. With ALMA survey observations of gaseous disks around hundreds, if not thousands, of young stars in the coming years, disk-based dynamical masses will become an industry, and the results will provide critical tests of pre-main-sequence stellar evolution models. This SMA study also highlights the fact that the smaller millimeter telescopes can coexist and thrive in the ALMA era, with a mix of innovative, highly focused programs 


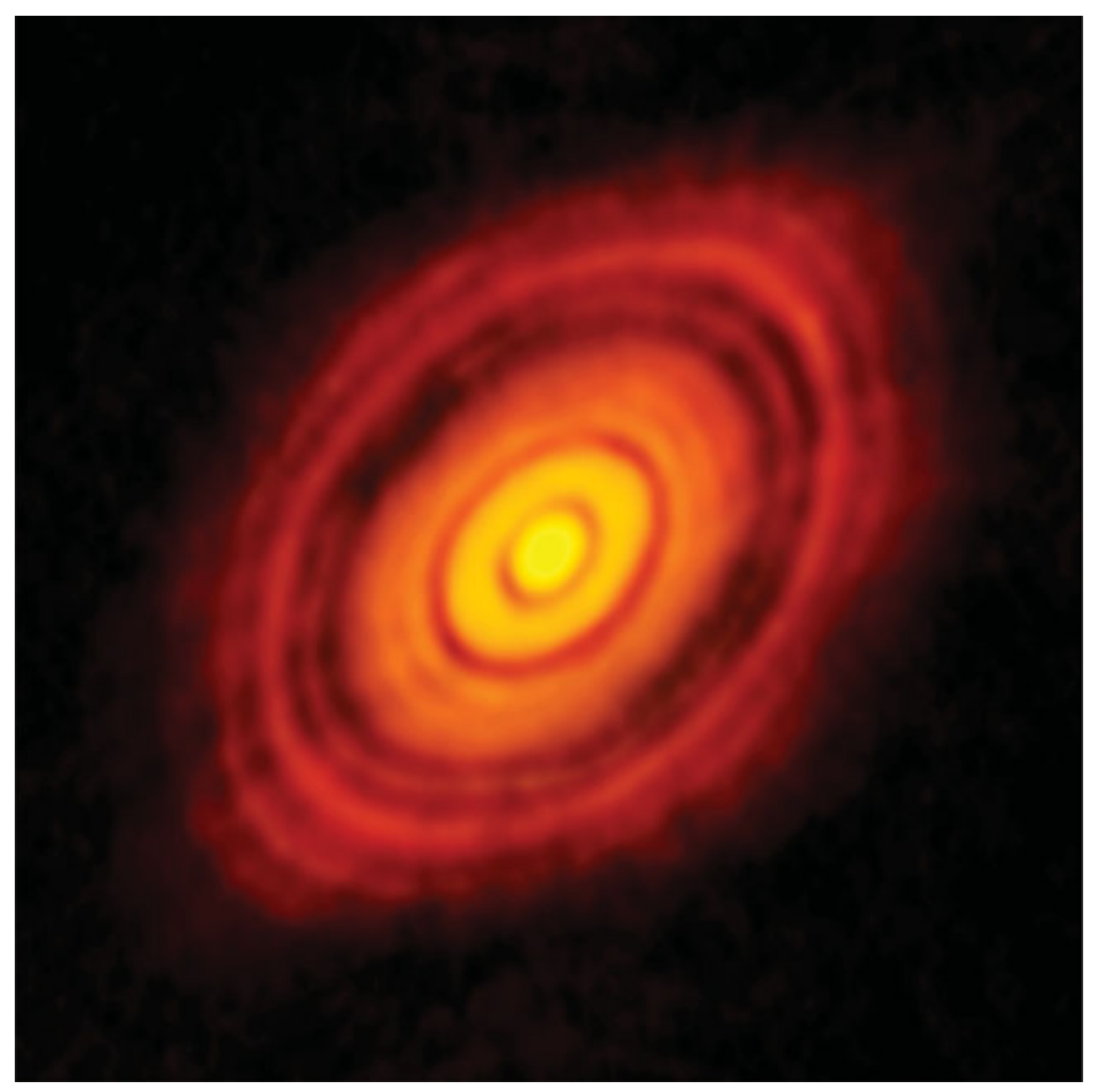

Figure 4. ALMA 1.3 millimeter continuum image of HL Tau, from the 05 November 2014 ALMA Press Release ("Revolutionary ALMA Image Reveals Planetary Genesis") at www. almaobservatory.org/press-room/press-releases/. The disk diameter is about 235 AU, and the beam size (35 milliarcseconds) is $5 \mathrm{AU}$.

and preparatory work (analogous to 2 meter class optical telescopes, or university based high performance computer clusters).

\section{ALMA's Revolutionary Angular Resolution}

The first results from the Long Baseline Campaign in fall 2014 as part of ALMA's Science Verification process are already remarkable, providing unprecedented angular resolution. Figure 4 shows the already iconic high resolution ALMA image of the disk surrounding the $<1$ Myr-old star HL Tau at 1.3 millimeters (ALMA Partnership et al. 2015). The time on source was 4.5 hours, using 25 to 30 antennas. The beam size is 35 milliarcseconds, corresponding to $5 \mathrm{AU}$. Most striking is the pattern of concentric bright and dark rings in the disk, tracing zones of concentrated and depleted solid particles. Some, or perhaps all, of the grooves may be carved out by the gravitational influence of giant planets in formation. Given the young age of this system, which is still partly embedded in an envelope, the formation of a system of planets is perhaps surprising. However, other explanations for these nested rings have been proposed, such as magnetohydrodynamic zonal flows and condensation fronts of volatiles, which must be considered and tested. Interestingly, the innermost regions of the HL Tau disk in this image show brightness temperatures in excess of $200 \mathrm{~K}$ and are almost certainly optically thick. To probe dust 
substructure in the zone of terrestrial planet formation in this system will require high resolution observations at longer (centimeter) wavelengths, to penetrate the inner disk column; no existing radio telescope has this capability.

What substructure will disks around other young stars show? As experience with models of the formation of our Solar System demonstrate, it is dangerous to extrapolate from a single example to all other systems. We really don't know what to expect. Will other disks also be comprised of nested rings, as seen in HL Tau? Giant planets have long been predicted to open gaps in disk gas, and particle trapping in the pressure maxima outside these gaps can make these features very prominent in millimeter continuum emission. Will we see spiral waves in disks, expected to be launched by these giant planets? Or will we see stochastic clumps, as solids are concentrated in turbulent eddies throughout the disk? The stars in Nearby Young Moving Groups will be crucial proving grounds for testing theoretical ideas about planet formation and disk-planet interaction, because of their proximity. For the TW Hya disk, for example, ALMA can resolve lowamplitude features as small as $2 \mathrm{AU}$, sufficient to measure planetary gaps on scales compatible with our Solar System architecture, and to probe turbulent fluctuations with sizes comparable to the gas pressure scale height over most of the disk, thereby providing stringent constraints.

\section{Concluding Remarks}

The ALMA era in millimeter astronomy is well underway, with revolutionary sensitivity, image fidelity, and high angular resolution. The early science results from ALMA on disks around young stars are tantalizing, unexpected, and incredibly exciting. We have a lot to look forward to.

\section{References}

Carpenter, J. M., Ricci, L., \& Isella, A. 2014, ApJ, 787, 42

Mathews, G. S., Williams, J. P., \& Ménard, F. 2012, ApJ, 753, 59

Menu, J., van Boekel, R., Henning, T., et al. 2014, A\& A, 564, A93

Partnership, A., Brogan, C. L., Perez, L. M., et al. 2015, arXiv:1503.02649

Rosenfeld, K. A., Andrews, S. M., Wilner, D. J., \& Stempels, H. C. 2012, ApJ, 759, 119

Zhang, K., Isella, A., Carpenter, J. M., \& Blake, G. A. 2014, ApJ, 791, 42 